\title{
El diagnóstico psicopedagógico para la inclusión socioeducativa de los escolares con necesidades educativas especiales
}

\section{The psychopedagogical diagnosis for the socio-educational inclusion of schoolchildren with special educational needs}

\author{
Pablo A. Martínez \\ Universidad Central "Marta Abreu" de Las Villas. Santa Clara. Villa Clara. Cuba \\ ORCID: https://orcid.org/0000-0001-5529-8199 \\ Lismay Pérez \\ Universidad Central "Marta Abreu” de Las Villas. Santa Clara. Villa Clara. Cuba \\ ORCID: https://orcid.org/0000-0002-6918-5408 \\ Marieta Alvarez \\ Universidad Central "Marta Abreu" de Las Villas. Santa Clara. Villa Clara. Cuba \\ ORCID: https://orcid.org/0000-0002-8775-4854
}

Received 03-12-20 Revised 05-25-20 Accepted 09-27-20 On line 09-29-20

*Correspondence

Email: pmmorales@uclv.cu
Cite as:

\footnotetext{
Martínez, P.A., Pérez, L., \& Alvarez, M. (2020). El diagnóstico psicopedagógico para la inclusión socioeducativa de los escolares con necesidades educativas especiales. Propósitos y Representaciones, 8 (SPE3), e735. Doi: http://dx.doi.org/10.20511/pyr2020.v8nSPE3.735
} 


\section{Resumen}

El presente artículo expone los resultados obtenidos como parte del trabajo desarrollado en una de las tareas del proyecto de investigación "La Pedagogía Especial como sustento para la inclusión socioeducativa de los escolares con necesidades educativas especiales (NEE)", dirigida a la elaboración de acciones didáctico metodológicas para la realización del diagnóstico psicopedagógico como sustento de la inclusión socioeducativa de los escolares con NEE. Desde una perspectiva cualitativa, considerando como grupos de enfoque a los docentes de instituciones de los diferentes niveles educativos de la ciudad de Santa Clara, provincia Villa Clara, Cuba, quienes participaron en un curso de entrenamiento impartido en el contexto del referido proyecto de investigación, se logró la elaboración, a partir del criterio colectivo, de acciones relativas a la preparación teórica y determinación de aspectos e indicadores que guíen el proceso de diagnóstico; recopilación de datos empíricos acerca del alumno y sus entornos; elaboración de la caracterización psicopedagógica del alumno; la construcción de la estrategia dirigida a favorecer la inclusión socioeducativa de cada alumno y las relativas al seguimiento y retroalimentación del proceso de diagnóstico psicopedagógico.

Palabras clave: Diagnóstico psicopedagógico; Inclusión socioeducativa; Necesidades educativas especiales

\section{Summary}

This article exposes the results obtained as part of the work carried out in one of the tasks of the research project "Special Pedagogy as a support for the socio-educational inclusion of schoolchildren with special educational needs (NEE)", aimed at the development of didactic actions methodologies for the realization of the psychopedagogical diagnosis as support for the socio-educational inclusion of schoolchildren with SEN. From a qualitative perspective, considering as focus groups the teachers of institutions of the different educational levels of the city of Santa Clara, Villa Clara province, Cuba, who participated in a training course given in the context of the aforementioned research project, the elaboration, based on the collective criteria, of actions related to theoretical preparation and determination of aspects and indicators that guide the diagnostic process was achieved; collection of empirical data about the student and their environments; elaboration of the psychopedagogical characterization of the student; the construction of the strategy aimed at favoring the socio-educational inclusion of each student and those related to the monitoring and feedback of the process of psychopedagogical diagnosis.

Keywords: Psychopedagogical Diagnosis; Socioeducational Inclusión; Special Educational Needs

\section{Introducción}

\section{El diagnóstico psicopedagógico}

Una de las tareas básicas de los docentes y especialistas que se involucran en la atención educativa de los escolares con necesidades educativas especiales (NEE) es la obtención de la información necesaria para conocerlos a ellos y a sus entornos. La más completa herramienta de que disponen en la actualidad con este fin es precisamente el diagnóstico, entendido como proceso, el cual constituye el punto de partida para el desarrollo de una labor educativa y de enseñanzaaprendizaje que asegure la inclusión socioeducativa de dichos escolares.

El tema del diagnóstico constituye un problema debatido por las ciencias sociales en la actualidad. La Pedagogía como ciencia de la Educación, lo utiliza para profundizar y transformar el estado educativo del escolar, en estrecho vínculo con los agentes sociales como potenciadores efectivos de su desarrollo. De esta forma se establece la relación entre la sociedad, la cultura y la educación como sistema, dirigida a lograr la inclusión del escolar como elemento activo y transformador (Akudovich et al., 2011). 
Indudablemente el contenido de este artículo no pretende abordar sustancialmente el tema del diagnóstico, mucho menos agotarlo. Los autores solo esclarecen la posición teórica que asumen para el cumplimiento de la correspondiente tarea de investigación en el contexto del proyecto "La Pedagogía Especial como sustento para la inclusión socioeducativa de los escolares con necesidades educativas especiales (NEE)".

Esclarecido este punto se está en condiciones de partir de los criterios expuestos por (Abreu, 1990), seguidor de los estudios de Vygotski, que propone tres etapas fundamentales por las que ha transitado el diagnóstico:

En una primera etapa estuvo basado en el uso exclusivo de test con fines clasificatorios, psicométricos (Binet, en Francia; Galton, en Inglaterra; Catell y otros, en Estados Unidos).

La segunda etapa, sustentado en el uso de entrevistas, autobiografías, estudio anamnésico, que permiten un conocimiento más global del sujeto y su evolución (Psicoanálisis).

En la tercera etapa el diagnóstico se elabora a través de la vía experimental, y está dirigido hacia áreas como los procesos cognoscitivos, reacciones del niño ante el juego, el aprendizaje.

En Cuba, la tendencia más generalizada en la actualidad es la integración de las tres corrientes que han caracterizado la evolución del diagnóstico por medio del trabajo interdisciplinario de diferentes especialistas.

A tono con esta tendencia se define el diagnóstico desde una perspectiva amplia como:

Una categoría general que se utiliza en todos los tipos y aspectos de la actividad humana; es decir, el ser humano, al analizar y evaluar los problemas y situaciones que constantemente surgen ante sí, trata de buscar sus posibles explicaciones, las causas y posibles soluciones, se enfrasca, de hecho, en un proceso de evaluación y diagnóstico, a través del cual se identifican las fuerzas motrices que lo producen, su génesis, particularidades y el curso del desarrollo alcanzado por un hecho o fenómeno dado y las posibilidades de su promoción a otros niveles de desarrollo. (Beatón, 1999, p. 128)

Desde esta concepción, el autor resalta como punto de partida la indagación y descubrimiento de las causas para la búsqueda de las soluciones a favor de la promoción del desarrollo.

Sin embargo, en la literatura sobre el tema y en la práctica educacional se constatan enfoques diferentes respecto a la definición del diagnóstico: diagnóstico psicológico, diagnóstico pedagógico o diagnóstico psicopedagógico.

En esta dirección varios autores definen el diagnóstico psicológico a partir de considerarlo: "un acto de gran importancia, ya que condiciona el tratamiento y evolución ulterior del sujeto. Exige del psicólogo no sólo maestría para aplicar las técnicas sino, además, una gran experiencia, una vasta cultura y mucha intuición" (Sillamy, 1967, p. 97).

Según Arias (1986):

... todo diagnóstico es el producto de un proceso, a través del cual se hace un análisis y una síntesis de la información que se posea y obtenga...éste se realiza para una intervención, promover un desarrollo y elevar la calidad de su vida. (p.128) 
Este autor señala, además, la necesidad de conocer la dinámica de la historia individual del desarrollo del sujeto, qué se ha producido y cómo ha devenido, en el proceso de formación que se está estudiando y es objeto de diagnóstico, así como, la necesidad no solo de descubrir los problemas o insuficiencias del desarrollo actual o real, sino el apreciar y llegar a conocer las posibilidades y reservas con que cuenta el sujeto, de sus potencialidades en su proceso de desarrollo ulterior.

Por otro lado, desde la Pedagogía la utilización es diversa, por ejemplo, se usa para determinar el rendimiento o aprovechamiento escolar. También para valorar los resultados de la atención diferenciada. Del mismo modo, y en un marco más general, permite evaluar el estado de planes y programas de la enseñanza en cualquier nivel. Similarmente puede ser utilizado en el análisis de la efectividad de determinados métodos de enseñanza y educación, y hasta en las propias estrategias y estilos de aprendizaje de los alumnos, factores todos que van encaminados a un enfoque personalizado y por lo tanto a una respuesta a la diversidad.

El diagnóstico pedagógico García (1995) lo define como:

Un tipo muy peculiar de evaluación que tiene en cuenta y se centra en las diferentes realidades que están implicadas en el proceso de enseñanza-aprendizaje de un alumno. Cinco serían, al menos, las variables más relevantes a las que ha de dirigir sus esfuerzos: Quién enseña (el profesor); a quién se enseña (o quién aprende, el alumno); lo que se enseña/aprende (programa/disciplina); cómo se enseña (metodología didáctica); el contexto y marco desde el que se educa y enseña (la sociedad, el sistema educativo, institución, grupo, curso, nivel, etapa. (p. 88)

En este planteamiento se iguala el diagnóstico a la evaluación, como corriente que se sigue en Latinoamérica. Se presta especial atención a los factores externos o ambientales que inciden en el niño y que pueden condicionar e incluso determinar, sus logros académicos. Se entiende como un proceso técnico, de constatación, identificación, interpretación y valoración de la interacción existente entre las variables más relevantes implicadas en el aprendizaje de un alumno o grupo para comprender o explicar su interacción (Pérez, Martínez, \& Carrera, 2016).

Desde otra perspectiva ha sido reconocido como el estudio profundo del estado de un proceso o producto de carácter pedagógico, que posibilita la identificación de logros, dificultades, potencialidades y sus causas, en función de un objetivo determinado, en un momento dado, con el propósito final de su transformación Silvestre (2003).

En esta definición no se aprecia la consideración del diagnóstico como proceso, tampoco se centra en el niño y sus entornos. define como:

El diagnóstico psicopedagógico se aborda también por varios autores. Álvarez (1998) lo

Proceso que lleva a la identificación de las necesidades específicas de cada individuo tomando en cuenta su entorno, que precisa las áreas débiles y las fuertes (necesidades y potencialidades) siendo un proceso continuo de evaluación-intervención con una retroalimentación continua de esta evolución y con un enfoque psicopedagógico. (p.13)

La autora citada desde la visión de proceso, particulariza en la detección de necesidades y potencialidades tanto del niño como de los contextos, dirige la atención a la retroalimentación dinámica y sistemática del desarrollo del niño con un enfoque psicopedagógico.

Nieves (2000) lo asume como:

El proceso de toma de decisiones, concebidas sobre la base del análisis y la valoración de un cúmulo de información conscientemente recopilada y cuyo objetivo es diseñar un programa de 
intervención que satisfaga las necesidades específicas sociales y académicas del menor. El conjunto inicial de decisiones posee un carácter tentativo y se va modificando en el proceso de intervención, en la medida en que se revelan nuevos hallazgos, a través del seguimiento y la evaluación de la eficacia del programa inicialmente concebido y paulatinamente enriquecido, rectificado y perfeccionado en el propio proceso de su ejecución. (p. 38)

La autora propone un modelo de diagnóstico desde la concepción de proceso, establece cada una de las etapas y pondera el diseño de la estrategia de atención educativa, aunque se enfoca más hacia el diagnóstico especializado que realizan los Centros de Diagnóstico y Orientación, sin llegar a profundizar en el que realiza la institución educativa.

Una vez planteadas las diferentes definiciones consultadas respecto al diagnóstico psicopedagógico, el análisis de las mismas conduce hacia algunas reflexiones:

No se aprecian contradicciones en cuanto a los rasgos que definen al diagnóstico psicopedagógico como proceso. En las definiciones de estos autores están presentes prácticamente los mismos elementos esenciales, se asumen en esta investigación:

- El carácter de proceso dinámico, individual y desarrollador.

- La determinación de las etapas o momentos:

- Elaboración y actualización de la caracterización psicopedagógica.

- Identificación de necesidades y potencialidades.

- Elaboración y actualización de la estrategia de atención educativa.

En este contexto, en la concepción actual del diagnóstico psicopedagógico para la atención educativa en Cuba, se reconocen tres dimensiones: el diagnóstico en la edad temprana y preescolar, el diagnóstico escolar y el diagnóstico especializado. Se definen objetivos específicos para cada una, además se precisa que no están contrapuestas, coexisten en estrecha relación dialéctica (Akudovich et al., 2011).

Esta visión multidimensional no es excluyente de la necesaria asunción del diagnóstico psicopedagógico como un proceso único e integral, sin embargo, se corre el riesgo, en su aplicación, de una fragmentación del proceso en las tres dimensiones que rompa su dialéctica interna, lo que dificultaría, en consecuencia, el cumplimiento de su carácter dinámico, continuo y sistemático.

En el diagnóstico en la edad temprana y preescolar, se identifica la caracterización psicopedagógica como valoración del desarrollo. El conocer mejor a los niños por parte del docente le permite estar más preparado para garantizar la atención individual y diferenciada, cumplir el principio del humanismo, manifestar amor y respeto hacia ellos y la preocupación por su educación y desarrollo.

La valoración del desarrollo presupone el dominio por los docentes de una concepción del desarrollo, el conocimiento de la periodización del desarrollo y las características psicológicas de cada una de las etapas; así como también de los métodos y las técnicas para la recogida de información. 
La evaluación realizada por los docentes, lleva a una valoración del desarrollo integral, que incluye lo biológico, lo psicológico y lo social, teniendo en cuenta los logros del desarrollo alcanzados, en correspondencia con la actividad fundamental de la edad. El criterio del colectivo garantiza una valoración objetiva del desarrollo de cada niño y permite además determinar los aspectos donde es necesario trabajar más.

Al concluir el curso escolar se efectúa la evaluación final del niño teniendo en cuenta como objetivo fundamental realizar una valoración general del desarrollo alcanzado por cada uno en particular y de todo el grupo en general.

El diagnóstico psicopedagógico tiene un fin educativo, formativo, no para arribar a conclusiones que identifiquen indefinidamente, que etiqueten o estigmaticen a los escolares, no para clasificarlos o igualarlos a otros, sino para elaborar las estrategias de atención educativa más oportunas que aseguren la igualdad de oportunidades y condiciones de éxito para el desarrollo de todos, es decir para asegurar su inclusión socioeducativa. Este proceso permite la identificación de limitaciones y dificultades, pero los más importante es que se descubren potencialidades, capacidades, reservas, que constituyen el punto de apoyo básico de una pedagogía humanista, integradora, normalizadora, de éxito para todos (Akudovich et al., 2011).

\section{La inclusión socioeducativa de los escolares con necesidades educativas especiales NEE}

El concepto de inclusión educativa aún no resulta bien comprendido y se encuentran variadas interpretaciones del mismo tanto en la literatura científica como en la práctica educativa.

La inclusión en el plano más estrecho pudiera verse como un concepto teórico de la pedagogía que hace referencia al modo en que la escuela debe dar respuesta a la diversidad, como también pudiera pensarse que es un concepto surgido en los años 90 que pretendió sustituir al de integración, el que por demás perdura de manera dominante aún en la práctica educativa. (Borges \& Orosco, 2013, p. 6)

En el informe referido al Estado Mundial de la Infancia (2013) se expone:

La preocupación por la inclusión se basa en el reconocimiento de que todos los niños y las niñas son miembros plenos de la sociedad; de que cada niño es un individuo único a quien asiste el derecho de que le respeten y le consulten; de que todos tienen habilidades y aspiraciones que vale la pena apoyar, así como necesidades que hay que satisfacer; y de que sus aportes deben ser valorados y promovidos. La inclusión requiere que la sociedad facilite el acceso a la infraestructura física, la información y los medios de comunicación, a fin de que todos los puedan utilizar; erradicar la discriminación con el objeto de que nadie se vea forzado a sufrirla; y brindar protección, apoyo y servicios para que todos los niños y niñas con discapacidad disfruten de sus derechos, al igual que todos los demás niños. (UNICEF, 2013, p. 3)

En Cuba la inclusión educativa es asumida teórica y metodológicamente desde la Pedagogía. Esto implica una visión sobre el modo en que la escuela debe dar respuesta a la diversidad. Su supuesto básico es que hay que modificar el sistema escolar para que responda a las necesidades de todos los alumnos, en vez de que sean los alumnos quienes deban adaptarse al sistema, integrándose a él (Borges \& Orosco, 2013).

En Cuba la inclusión educativa es entendida como una concepción que reconoce el derecho de todos a una educación de calidad, con independencia de sus particularidades y características que determinan las peculiaridades en su desarrollo y que propicie su integración a la sociedad como individuos plenos en condiciones de poder disfrutar las posibilidades que esta ofrece y contribuir a su perfeccionamiento. (Borges \& Orosco, 2013, p. 7) 
En diferentes fuentes consultadas se aprecian limitaciones en cuanto a la definición del término inclusión socioeducativa. En el informe referido al Estado Mundial de la Infancia (2013) se exponen criterios que pueden contribuir al establecimiento de una definición al respecto:

La inclusión beneficia a todo el mundo. (...) un programa de estudios inclusivo -es decir, centrado en el niño y con representaciones de personas con impedimentos para reflejar un sector real de la sociedad- puede ampliar tanto los horizontes de los niños que, de otra manera, podrían ver limitadas sus ambiciones y opciones a causa de sus discapacidades, como los de los niños sin discapacidad, que aprenderían a valorar la diversidad, las habilidades y la preparación necesarias para construir una sociedad inclusiva para todos. Cuando los logros educativos llevan a la consecución de trabajo u otros medios para ganarse la vida, el niño con discapacidad puede avanzar y asumir su lugar como miembro pleno y en igualdad de condiciones del mundo adulto, un miembro que produce y consume. (UNICEF, 2013, p. 3)

Los análisis descritos con anterioridad permiten aproximarse a una definición de inclusión socioeducativa en correspondencia con las concepciones de los autores anteriormente citados. Por tanto, el colectivo de investigadores del proyecto de investigación "La Pedagogía Especial como sustento para la inclusión socioeducativa de los escolares con necesidades educativas especiales (NEE)" propone:

La inclusión socioeducativa constituye un proceso dinámico y multifactorial que promueve la igualdad de oportunidades para el acceso, la permanencia, el aprendizaje, el éxito escolar y la preparación para la vida de todos los alumnos, a través de los agentes socializadores, en aras de alcanzar un papel protagónico en su desarrollo personal y social. (Especial, 2016, p. 6)

A modo de resumen, se asume la inclusión socioeducativa como un proceso, ya que transcurre simultáneamente en correspondencia con las etapas del desarrollo de la personalidad del individuo, incidiendo de manera dinámica en su formación y transformación los más diversos factores externos e internos que aportan influencias significativas facilitadoras de la igualdad de oportunidades para el acceso y la permanencia en un sistema educativo dado.

La presencia de agentes socializadores se convierte en una premisa para la concepción de una educación de calidad que no solo se expresa en la adquisición de conocimientos sino también en el despliegue de hábitos, habilidades, capacidades que le permitan al sujeto ser protagónico dentro de la sociedad independientemente de su individualidad, condición, necesidad educativa especial.

De la definición anterior puede reconocerse que la inclusión socioeducativa se orienta hacia la ejecución de acciones en dos importantes planos de influencia:

- El primero relacionado con las acciones que propicien el mejoramiento de la calidad del proceso pedagógico que se desarrolla en los diferentes escenarios educativos, de modo que se promueva -al máximo posible- relaciones afectuosas, entornos de comunicación asertiva y el fortalecimiento de valores como la tolerancia, el colectivismo y el respeto a las diferencias.

- El segundo se relaciona con la visión que deben asumir los centros educativos para prever las acciones y las estrategias necesarias y suficientes para garantizar la preparación socio laboral de todos los alumnos, con énfasis en aquellos que manifiestan necesidades educativas especiales.

Una vez expuestos los criterios teóricos que asumen los autores del artículo respecto al diagnóstico psicopedagógico y a la inclusión socioeducativa de los escolares con necesidades educativas especiales, se está en condiciones de declarar la interrogante de partida del proceso 
investigativo desarrollado, como parte de la tarea relativa al diagnóstico psicopedagógico en el contexto del proyecto "La Pedagogía Especial como sustento para la inclusión socioeducativa de los escolares con necesidades educativas especiales (NEE)”, en este caso: ¿Cuáles serán las acciones didáctico metodológicas que deben desplegar los docentes para realizar el diagnóstico psicopedagógico para la inclusión socioeducativa de los escolares con necesidades educativas especiales (NEE)?

El presente artículo pretende la exposición de los resultados del proceso investigativo dirigido a elaborar acciones didáctico metodológicas para la realización del diagnóstico psicopedagógico como sustento de la inclusión socioeducativa de los escolares con NEE.

\section{Método}

El proceso llevado a cabo siguió una perspectiva cualitativa y se enmarcó durante la realización de un curso de entrenamiento conducido por los autores y otros investigadores del referido proyecto de investigación durante los dos últimos cursos escolares en instituciones de la ciudad de Santa Clara, Villa Clara, Cuba, pertenecientes a los diferentes niveles educativos. Se organizaron grupos de enfoque (Sampieri, Collado, \& Lucio, 2014) con los docentes de cada institución participantes en el curso. La muestra quedó conformada de la siguiente manera:

\section{Tabla 1.}

Grupos de enfoque

\begin{tabular}{cc}
\hline $\begin{array}{c}\text { Tipo de institución por nivel } \\
\text { educativo }\end{array}$ & $\begin{array}{c}\text { Cantidad de } \\
\text { participantes }\end{array}$ \\
\hline Círculo Infantil & 12 \\
Escuela primaria & 21 \\
Escuela especial & 10 \\
Secundaria básica & 7 \\
Preuniversitario & 12 \\
Facultad universitaria & 22 \\
TOTALES & 84 \\
\hline
\end{tabular}

Las sesiones de discusión fueron realizadas al terminar el análisis de los diferentes contenidos relativos al diagnóstico psicopedagógico como sustento para la inclusión socioeducativa de los escolares con NEE previstos en el programa del curso de entrenamiento (Martínez, Alvarez, Pérez, \& Hernández, 2017), para ello se planteó la siguiente guía de temas para la discusión:

- Aspectos e indicadores a tener en cuenta para el diagnóstico psicopedagógico como sustento para la inclusión socioeducativa de los escolares con NEE.

- Procedimiento para la recopilación de datos empíricos acerca del alumno y sus entornos. 
- Procedimiento para la elaboración de la caracterización psicopedagógica del alumno.

- Acciones a tener en cuenta para la construcción de la estrategia dirigida a favorecer la inclusión educativa de cada alumno.

- Realización del seguimiento y retroalimentación del proceso de diagnóstico psicopedagógico como sustento para la inclusión socioeducativa de los escolares con NEE.

\section{Resultados}

Después de realizado el curso de entrenamiento, en cada institución educativa se procedió a organizar los grupos de enfoque con los docentes participantes. En estos grupos la discusión fue conducida a partir de la referida guía de temas. Seguidamente se presentan, de manera resumida, los resultados de los análisis realizados.

En la institución de educación preescolar participaron 12 docentes (un directivo, cinco educadoras y seis auxiliares pedagógicas). Respecto al primer tema de la guía referente a los aspectos e indicadores a tener en cuenta para el diagnóstico psicopedagógico como sustento para la inclusión socioeducativa de los escolares con NEE proponen que no deben faltar la psicomotricidad, las relaciones interpersonales, los tipos de inteligencia, las potencialidades creativas, la comunicación, los estados afectivos, el grupo, la institución educativa, la familia y los factores socioeconómicos y culturales de la comunidad.

En el caso del proceder para la elaboración de la caracterización psicopedagógica del alumno coinciden en afirmar la importancia de la selección de métodos y técnicas, su aplicación y el análisis de cada instrumento.

Respecto al tema referido a los procedimientos para la elaboración de la caracterización psicopedagógica del alumno las opiniones convergen en afirmar que presupone una valoración con enfoque objetivo, integral, explicativo, individualizado y desarrollador, que es una valoración integral del niño que permite la determinación de potencialidades y necesidades del desarrollo del alumno y entornos. Se manifiestan, además, en la seriedad y profesionalidad para la redacción del informe.

Sobre las acciones a tener en cuenta para la construcción de la estrategia dirigida a favorecer la inclusión educativa de cada alumno coinciden en afirmar que las acciones se proyectan a partir de las necesidades y potencialidades del desarrollo de los alumnos y los entornos sociales en que se desenvuelven, es decir la familia, el grupo, la escuela y la comunidad.

En cuanto a la realización del seguimiento y retroalimentación del proceso de diagnóstico psicopedagógico como sustento para la inclusión socioeducativa de los escolares con NEE, proponen la atención sistemática a estos alumnos, el seguimiento a su desempeño, la valoración de las acciones ejecutadas y la reelaboración o propuesta de nuevas acciones educativas en correspondencia con el desarrollo de la de los alumnos constatado y la marcha del proceso de inclusión.

En la institución de educación primaria participaron 21 docentes (dos directivos, 10 maestros, cinco auxiliares pedagógicas y cuatro especialistas).

Comienza la sesión de trabajo con la presentación de los profesores que conducirán el debate y la propuesta del proceder para el trabajo en el grupo. Sobre los aspectos e indicadores a 
tener en cuenta para el diagnóstico psicopedagógico como sustento para la inclusión socioeducativa de los escolares con NEE, los docentes emiten diversas opiniones argumentando en cada caso su propuesta, de manera general coinciden en proponer como aspectos a considerar en el diagnóstico psicopedagógico como sustento para la inclusión socioeducativa de los escolares con NEE la consulta de la bibliografía especializada respecto a la inclusión educativa, el proceso de diagnóstico psicopedagógico y las características de la personalidad del alumno, la psicomotricidad, las relaciones interpersonales, los estilos de aprendizaje, los tipos de inteligencia, las motivaciones y preferencias, la zona de Desarrollo Próximo, la resiliencia, los estados afectivos, el desarrollo volitivo, el grupo, la institución educativa, la familia y la comunidad. Como respuestas al proceder para la recopilación de datos empíricos acerca del alumno y sus entornos, en algunos casos mencionan la determinación de métodos y técnicas de investigación y sus instrumentos correspondientes y la aplicación de los mismos, en su mayoría agregan además a los anteriores, el análisis de los aspectos recopilados.

En el debate, sobre el proceder para la elaboración de la caracterización psicopedagógica del alumno los participantes mencionan la valoración integral de los resultados obtenidos con enfoque objetivo, integral, explicativo, individualizado y desarrollador, la determinación de potencialidades y necesidades del desarrollo del alumno y sus entornos, resaltando la importancia de la redacción del informe de caracterización psicopedagógica.

Acerca de las acciones a tener en cuenta para la construcción de la estrategia dirigida a favorecer la inclusión educativa de cada alumno los criterios se enmarcan en la elaboración de acciones educativas a partir de las necesidades y potencialidades del desarrollo de los alumnos y los entornos sociales, así como la selección de los recursos necesarios para la implementación de las acciones.

Los docentes coinciden en reconocer cómo el proceder para la realización del seguimiento y retroalimentación del proceso de diagnóstico psicopedagógico como sustento para la inclusión socioeducativa de los alumnos con NEE la recopilación sistemática de datos empíricos acerca del alumno y sus entornos, el análisis y valoración de los datos recopilados, la valoración del cumplimiento de las acciones elaboradas así como la reelaboración o propuesta de nuevas acciones según la marcha del proceso de inclusión.

La sesión de trabajo desarrollada en la institución de educación especial comenzó con la interpretación de frases referidas a la inclusión de los niños con NEE donde los docentes reconocieron la importancia y actualidad del tema, así como la necesidad de superación en el mismo. Asistieron ocho maestros, un logopeda y un profesor de Educación Física.

Los participantes opinaron acerca de los aspectos e indicadores a tener en cuenta para el diagnóstico psicopedagógico como sustento para la inclusión socioeducativa de los escolares con NEE llegando al consenso, de que no debían obviarse la psicomotricidad, las relaciones interpersonales, los estilos de aprendizaje, los tipos de inteligencia, las potencialidades creativas, las motivaciones y preferencias, la Zona de Desarrollo Próximo, la comunicación, la resiliencia, los estados afectivos, el desarrollo volitivo, los intereses laborales, el grupo, la institución educativa, la familia y la comunidad.

Sobre el procedimiento para la recopilación de datos empíricos acerca del alumno y sus entornos los criterios coinciden en proponer como acciones la determinación de métodos y técnicas, la elaboración o selección de los instrumentos correspondientes, la aplicación de métodos y técnicas, el análisis de la aplicación, solo la logopeda sugiere el procesamiento de los datos recopilados.

Las opiniones en torno a la elaboración de la caracterización psicopedagógica del alumno los docentes resaltan la redacción del informe como elemento fundamental que debe reflejar la 
valoración integral de los resultados obtenidos en la aplicación de los métodos técnicas e instrumentos y propiciar la determinación de potencialidades y necesidades del desarrollo del alumno y sus entornos.

Al verter las opiniones sobre las acciones a tener en cuenta para la construcción de la estrategia dirigida a favorecer la inclusión educativa de cada alumno, los docentes proponen la elaboración de acciones educativas a partir de las necesidades y potencialidades del desarrollo de los alumnos y los entornos y la selección de los medios para la implementación de las acciones.

Sobre la realización del seguimiento y retroalimentación del proceso de diagnóstico psicopedagógico como sustento para la inclusión socioeducativa de los escolares con NEE los docentes proponen como procedimientos la recopilación sistemática de datos acerca del alumno y sus entornos, el análisis y valoración de los datos recopilados y la valoración del cumplimiento de las acciones educativas elaboradas.

En la institución de secundaria básica participaron siete docentes que imparten las asignaturas Educación Cívica, Química, Lengua Española, Matemática e Historia. Se inicia la sesión de trabajo con una breve explicación de la metodología a seguir para trabajar en los grupos de enfoque.

En cuanto a los aspectos e indicadores a tener en cuenta para el diagnóstico psicopedagógico como sustento para la inclusión socioeducativa de los escolares con NEE proponen que no deben faltar las relaciones interpersonales, los tipos de inteligencia, las potencialidades creativas, la comunicación, los estados afectivos, el grupo, la institución educativa, la familia y los factores socioeconómicos y culturales de la comunidad.

Sobre el proceder para la elaboración de la caracterización psicopedagógica del alumno coinciden en afirmar la importancia de la selección de métodos y técnicas, su aplicación, el análisis de cada instrumento y el procesamiento de la información obtenida.

Respecto a los procedimientos para la elaboración de la caracterización psicopedagógica del alumno las opiniones convergen en afirmar el enfoque objetivo, integral, explicativo, individualizado y desarrollador, que es una valoración integral del niño que permite la determinación de potencialidades y necesidades del desarrollo del alumno y entornos.

Acerca de las acciones a tener en cuenta para la construcción de la estrategia dirigida a favorecer la inclusión educativa de cada alumno refieren en su mayoría que las acciones se proyectan a partir de las necesidades y potencialidades del desarrollo de los alumnos y los entornos sociales en que se desenvuelven, es decir la familia, el grupo, la escuela y la comunidad.

Sobre los procedimientos para la realización del seguimiento y retroalimentación del proceso de diagnóstico psicopedagógico como sustento para la inclusión socioeducativa de los escolares con NEE, los docentes proponen la atención sistemática a estos alumnos, el seguimiento, la valoración de las acciones ejecutadas y la reelaboración o propuesta de nuevas acciones educativas en correspondencia con el desarrollo de la de los alumnos.

En la institución de educación preuniversitaria el grupo estuvo integrado por 12 docentes (un directivo y 11 profesores de las asignaturas Matemática, Historia, Lengua Española, Cultura Política, Química y Física). 
Al iniciar la sesión de trabajo se reconoció el apoyo de directivos y la preparación de los docentes en las acciones desarrolladas como parte de la superación profesional para la atención a alumnos con NEE.

En el primer momento los participantes opinaron acerca de los aspectos e indicadores a tener en cuenta para el diagnóstico psicopedagógico como sustento para la inclusión socioeducativa de los escolares con NEE llegando al consenso, de que no debían obviarse las relaciones interpersonales, los estilos de aprendizaje, los tipos de inteligencia, las potencialidades creativas, las motivaciones y preferencias, la Zona de Desarrollo Próximo, la comunicación, la resiliencia, los estados afectivos, el desarrollo volitivo, los intereses laborales, el grupo, la institución educativa, la familia y la comunidad.

Respecto al procedimiento para la recopilación de datos empíricos acerca del alumno y sus entornos los criterios estuvieron en torno a la determinación de métodos y técnicas, la elaboración o selección de los instrumentos correspondientes, la aplicación de métodos y técnicas y el análisis de la aplicación.

En cuanto a la elaboración de la caracterización psicopedagógica del alumno los docentes resaltan la redacción del informe como elemento fundamental que debe reflejar la valoración integral de los resultados obtenidos en la aplicación de los métodos técnicas e instrumentos y propiciar la determinación de potencialidades y necesidades del desarrollo del alumno y sus entornos.

Al emitir opiniones sobre las acciones a tener en cuenta para la construcción de la estrategia dirigida a favorecer la inclusión educativa de cada alumno, los docentes proponen la elaboración de acciones educativas a partir de las necesidades y potencialidades del desarrollo de los alumnos y los entornos sociales y la selección de los medios y recursos necesarios para la implementación de las acciones.

En el debate referido la realización del seguimiento y retroalimentación del proceso de diagnóstico psicopedagógico como sustento para la inclusión socioeducativa de los escolares con NEE los docentes coinciden en señalar como procedimientos la recopilación sistemática de datos acerca del alumno y sus entornos, el análisis y valoración de los datos recopilados y la valoración del cumplimiento de las acciones educativas elaboradas.

A la sesión de trabajo desarrollada en la facultad universitaria asistieron 22 profesores, entre ellos vicedecano de formación del profesional, coordinadores de carrera, profesores principales de año, profesores guías y profesores de las asignaturas. Comenzó con la interpretación de frases referidas a la inclusión de las personas con NEE y se explicó el proceder metodológico a seguir en la sesión de trabajo.

Los participantes coinciden en sus criterios que los aspectos e indicadores a tener en cuenta para el diagnóstico psicopedagógico como sustento para la inclusión socioeducativa de las personas con NEE que no deben faltar son las relaciones interpersonales, los estilos de aprendizaje, los tipos de inteligencia, las potencialidades creativas, las motivaciones y preferencias, la Zona de Desarrollo Próximo, la comunicación, la resiliencia, los estados afectivos, el desarrollo volitivo, los intereses laborales, el grupo, la institución educativa, la familia y la comunidad.

Sobre el procedimiento para la recopilación de datos empíricos acerca del alumno y sus entornos proponen la determinación de métodos y técnicas, la elaboración o selección de los instrumentos correspondientes, la aplicación de métodos y técnicas, el análisis de la aplicación, así como el procesamiento de los datos recopilados. 
Las opiniones en torno a la elaboración de la caracterización psicopedagógica del estudiante los docentes resaltan la redacción del informe como elemento fundamental que debe reflejar la valoración integral de los resultados obtenidos en la aplicación de los métodos técnicas e instrumentos y propiciar la determinación de potencialidades y necesidades del desarrollo del alumno y sus entornos.

Al referirse a las acciones a tener en cuenta para la construcción de la estrategia dirigida a favorecer la inclusión educativa de cada alumno, los profesores señalan la elaboración de acciones educativas a partir de las necesidades y potencialidades del desarrollo de los alumnos y los entornos y la selección de los recursos y medios para la implementación de las acciones.

Sobre la realización del seguimiento y retroalimentación del proceso de diagnóstico psicopedagógico como sustento para la inclusión socioeducativa de las personas con NEE coinciden en proponen como procedimientos la recopilación sistemática de datos acerca del alumno y sus entornos, el análisis y valoración de los datos recopilados y la valoración del cumplimiento de las acciones educativas elaboradas así como la reelaboración o propuesta de nuevas acciones educativas en correspondencia con el desarrollo de la personalidad de los alumnos y la marcha del proceso de inclusión.

Culminadas las diferentes sesiones de discusión en cada uno de los grupos de enfoque, se estuvo en condiciones de elaborar la propuesta de acciones didáctico metodológicas para la realización del diagnóstico psicopedagógico como sustento para la inclusión socioeducativa de los escolares con necesidades educativas especiales (NEE), tal como se exponen seguidamente.

Acciones didáctico metodológicas (Pérez, Martínez, \& Alvarez, 2019):

Acción 1: Preparación teórica y determinación de aspectos e indicadores que guíen el proceso de diagnóstico.

Objetivo: Garantizar la autopreparación del docente para la determinación de los aspectos e indicadores para el diagnóstico.

Operaciones:

- Consulta de la bibliografía especializada respecto a la inclusión educativa, el proceso de diagnóstico psicopedagógico y las características de la personalidad del alumno según la etapa del desarrollo ontogenético.

- Determinación de los aspectos e indicadores para el diagnóstico de la personalidad y los entornos del alumno. En este caso se sugiere particularizar en los siguientes aspectos:

- Psicomotricidad.

- Relaciones interpersonales.

- Estilos de aprendizaje.

- Tipos de inteligencia.

- Potencialidades creativas. 
- Motivaciones y preferencias.

- Zona de Desarrollo Próximo.

- Comunicación.

- Resiliencia.

- Estados afectivos.

- Desarrollo volitivo.

- Intereses laborales.

- Grupo.

- Institución educativa.

- Familia.

- Factores socioeconómicos y culturales de la comunidad.

Acción 2: Recopilación de datos empíricos acerca del alumno y sus entornos.

Objetivo: Recopilar los datos correspondientes a los aspectos e indicadores del desarrollo de la personalidad del alumno, así como de sus entornos.

Operaciones:

- Determinación de métodos y técnicas de investigación.

- Elaboración o selección de los instrumentos correspondientes.

- Aplicación de métodos, técnicas e instrumentos.

- Análisis y procesamiento de los datos recopilados.

Acción 3: Elaboración de la caracterización psicopedagógica del alumno.

Objetivo: Valorar el desarrollo del alumno en relación con sus entornos.

Operaciones:

- Valoración integral de los resultados obtenidos con enfoque objetivo, integral, explicativo, individualizado y desarrollador.

- Determinación de potencialidades y necesidades del desarrollo del alumno, las causas o factores que intervienen estimulando o entorpeciendo dicho desarrollo, así como de las fortalezas de los entornos sociales donde este se desenvuelve.

- Redacción del informe de caracterización psicopedagógica. 
Acción 4: Propuesta de acciones para la construcción de la estrategia dirigida a favorecer la inclusión educativa de cada alumno.

Objetivo: Elaborar acciones educativas, en correspondencia con las potencialidades y necesidades del desarrollo del alumno, así como las fortalezas de sus entornos.

Operaciones:

- Elaboración de acciones educativas a partir de las necesidades y potencialidades del desarrollo de los alumnos.

- Elaboración de acciones educativas a partir de las fortalezas de los entornos sociales en que se desenvuelven los alumnos (familia, escuela, grupo, comunidad).

- Selección de los medios y recursos necesarios para la implementación de las acciones educativas.

Acción 5: Seguimiento y retroalimentación del proceso de diagnóstico psicopedagógico.

Objetivo: Actualizar el proceso de diagnóstico psicopedagógico.

Operaciones:

- Recopilación sistemática de datos empíricos acerca del alumno y sus entornos.

- Análisis y valoración de los datos recopilados.

- Valoración del cumplimiento de las acciones educativas elaboradas.

- Reelaboración o propuesta de nuevas acciones educativas en correspondencia con el desarrollo de la personalidad de los alumnos constatado y la marcha del proceso de inclusión.

\section{Discusión}

El proceso investigativo llevado a cabo permitió satisfacer el objetivo inicialmente planteado por los autores, quedando elaborada una propuesta de acciones didáctico metodológicas para la realización del diagnóstico psicopedagógico como sustento para la inclusión socioeducativa de los escolares con necesidades educativas especiales (NEE), coherente con las concepciones que sobre el diagnóstico como proceso se han venido desarrollando desde el quehacer científico y en la práctica educativa en Cuba (Abreu, 1990) (Álvarez, 1998) (Beatón, 1999) (Nieves, 2000) (Akudovich et al., 2011).

Partiendo de estas concepciones generales sobre el diagnóstico las acciones didáctico metodológicas elaboradas sintetizan, o más bien hacen énfasis en aquellas que no deben dejar de desarrollar los docentes de cualquier tipo de institución educativa, como punto de partida para la modelación y puesta en práctica de estrategias que aseguren una verdadera inclusión de los escolares con necesidades educativas especiales, tanto en lo educativo, como en la vida social y laboral futura (Borges \& Orosco, 2013) (Akudovich et al., 2011) (Especial, 2016). 
El diagnóstico para la inclusión educativa es un proceso constante y dinámico, que ocurre como parte indispensable del proceso de formación de la personalidad de los escolares, donde docentes, familias y demás agentes educativos interactúan para comprender la forma peculiar en que el desarrollo traza su curso en cada niño particular, y descubre las formas más efectivas para estimularlo. De esta manera no es una evaluación de un niño, sino el estudio de la situación social en la que ocurre su desarrollo, entiéndase la consideración también de sus contextos, grupo, escuela, familia y comunidad.

La finalidad del diagnóstico para la inclusión radica en asegurar la efectividad del proceso de educación de calidad para todos, donde no se establecen barreras, ni diferencias, ni límites, sino que se buscan formas cooperativas, interactivas y cada vez más desarrolladoras para todos por igual.

Para enfrentar el proceso de diagnóstico para la inclusión socioeducativa de los escolares con necesidades educativas especiales, el docente, desde una sensibilización y compromiso con ese empeño; debe ser capaz de adquirir una preparación elevada en el tema y por eso en el documento se parte de una acción y operaciones relacionadas con este aspecto, donde a partir de la preparación teórica se sugieren indicadores que deben guiar este proceso. Dentro de ellos queremos significar, los que a nuestro juicio son imprescindible en el empeño de conducir un proceso educativo tendiente a la inclusión de los niños y adolescentes con necesidades educativas especiales:

Los tipos de inteligencias, considerando que la misma, es la capacidad de aprender y beneficiarse de la experiencia, de adaptarse a diversas situaciones y contextos, hay muchas formas de ser inteligente, todo individuo puede desarrollar cada inteligencia hasta un nivel adecuado de sus competencias, existen interacciones de desarrollo entre las mismas y cada una propone metas para impulsar el mismo, por lo que se deben descubrir las fortalezas particulares de cada educando para promover hasta el máximo posible, su inteligencia, a fin de hacerlos aptos y competentes no solo en la escuela, sino sobre todo en la vida. La resiliencia, capacidad que puede desarrollar todo ser humano para poder adaptarse y superar la adversidad. El desarrollo volitivo para precisar la disposición a la realización de esfuerzos (constancia), independencia en la toma de decisiones, aceptación de metas. Planteamiento independiente de metas y el control de reacciones y conductas inadecuadas y establecer también el comportamiento de las cualidades volitivas autodominio y perseverancia. En el grupo, los tipos sociométricos (Pérez et al., 2019).

Puede concluirse entonces que el diagnóstico psicopedagógico que se desarrolle en cualquier tipo de institución educativa por parte de sus docentes, como punto de partida para la inclusión socioeducativa de los escolares con necesidades educativas especiales, debe ser concebido como un proceso riguroso, sistemático, objetivo y dinámico, que parta de una adecuada determinación de indicadores, la correspondiente selección de métodos y técnicas con los correspondientes instrumentos de recogida de información sobre las particularidades de los escolares y sus entornos, que permita la valoración objetiva de su desarrollo y en consecuencia las correspondientes estrategias que aseguren el proceso inclusivo. Dicho proceso exige el constante seguimiento y actualización, en consonancia con la evolución del proceso inclusivo y de los escolares en él inmersos.

\section{Referencias}

Abreu, E. (1990). Diagnóstico de las desviaciones en el desarrollo psíquico. La Habana: Editorial Pueblo y Educación.

Akudovich, S. A., Cruz, C. R. Z., Rivero, M. L. N., Valdespino, J. B., Villavicencio, P. M., \& Acosta, N. H. (2011). El proceso de diagnóstico para la atención educativa. Bases 
metodológicas para la práctica (pp. 61). La Habana: Sello Editor EDUCACIÓN CUBANA.

Álvarez, C. (1998). Diagnóstico y zona de desarrollo próximo. Alternativa en la validación de una metódica del cuarto excluido. (Tesis en opción al grado científico de Doctor en Ciencias Pedagógicas), Instituto Central de Ciencias Pedagógicas, La Habana.

Beatón, G. A. (1999). Educación, desarrollo, evaluación y diagnóstico desde el enfoque histórico cultural. Ciudad de La Habana.

Borges, S., \& Orosco, M. (2013). Educación especial y educación inclusiva: un horizonte singular y diverso para igualar las oportunidades de desarrollo. La Habana: Sello Editor Educación Cubana.

Especial, D. d. E. (2016). Manual de acciones didácticas-metodológicas para la inclusión socioeducativas de los escolares con necesidades educativas especiales. Educación Especial. Universidad Central "Marta Abreu" de Las Villas. Santa Clara.

García, N. (1995). El diagnóstico pedagógico en la educación infantil. Revista Complutense de Educación, 6 (1), 74-100.

Martínez, P. A., Alvarez, M., Pérez, L., \& Hernández, L. M. (2017). El diagnóstico psicopedagógico para la inclusión socioeducativa de los escolares con necesidades educativas especiales. Orientaciones a los docentes. Educación Especial. Universidad Central "Marta Abreu" de Las Villas. Santa Clara.

Nieves, M. L. (2000). El diagnóstico como proceso de evaluación intervención: una nueva concepción Educación de alumnos con necesidades educativas especiales. Fundamentos y actualidad. Ciudad de La Habana: Editorial Pueblo y Educación.

Pérez, L., Martínez, P. A., \& Alvarez, M. (2019). Acciones didáctico metodológicas para el diagnóstico psicopedagógico como sustento para la inclusión socioeducativa de los escolares con NEE. Educación Especial. Universidad Central "Marta Abreu" de Las Villas. Santa Clara.

Pérez, L., Martínez, P. A., \& Carrera, M. (2016, Julio - Diciembre). El diagnóstico de la preparación para la vida escolar de los niños con discapacidad intelectual. Revista Científica Ra Ximhai. Paz, Interculturalidad y Democracia, 12 Número 5, 10.

Sampieri, R. H., Collado, C. F., \& Lucio, P. B. (2014). Metodología de la investigación. Sexta Edición (Sexta Edición ed.). México D. F.: McGRAW-HILL / INTERAMERICANA EDITORES, S.A. DE C.V.

Silvestre, M. (2003). El diagnóstico del proceso de enseñanza-aprendizaje. ICCP. La Habana.

Sillamy, N. (Ed.) (1967) Dictionnaire de la Psychologie. París: Larousse.

UNICEF. (2013). Estado mundial de la infancia 2013. Niños y niñas con discapacidad (pp. 164). Nueva York. 
